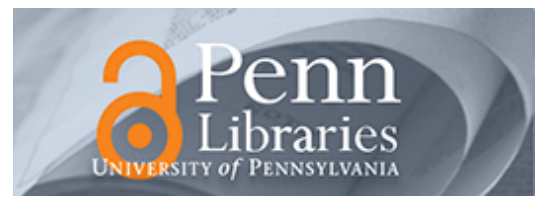

University of Pennsylvania

ScholarlyCommons

6-10-2010

\title{
Hydrodynamic Irreversibility in Particle Suspensions with Nonuniform Strain
}

Jeffrey S. Guasto

Haverford College

Andrew S. Ross

Haverford College

Jerry P. Gollub

University of Pennsylvania, Haverford College

Follow this and additional works at: https://repository.upenn.edu/physics_papers

Part of the Physics Commons

\section{Recommended Citation}

Guasto, J. S., Ross, A. S., \& Gollub, J. P. (2010). Hydrodynamic Irreversibility in Particle Suspensions with Nonuniform Strain. Retrieved from https://repository.upenn.edu/physics_papers/18

Suggested Citation:

Guasto, J.S., A.S. Ross and J.P. Gollub. (2010). "Hydrodynamic irreversibility in particle suspensions with nonuniform strain." Physical Review E 81, 061401.

(C) The American Physical Society

http://dx.doi.org/10.1103/PhysRevE.81.061401

This paper is posted at ScholarlyCommons. https://repository.upenn.edu/physics_papers/18

For more information, please contact repository@pobox.upenn.edu. 


\title{
Hydrodynamic Irreversibility in Particle Suspensions with Nonuniform Strain
}

\author{
Abstract \\ A dynamical phase transition from reversible to irreversible behavior occurs when particle suspensions \\ are subjected to uniform oscillatory shear, even in the Stokes flow limit. We consider a more general \\ situation with nonuniform strain (e.g., oscillatory channel flow), which is observed to exhibit markedly \\ different dynamics. The onset of irreversibility is delayed, and occurs simultaneously across the entire \\ channel. This behavior is only partially explained by self-organization and shear-induced migration. The \\ onset is accompanied by long-range correlated particle motion even at the channel center, where the \\ strain is negligible; this motion prevents the system from evolving into a reversible state. \\ Disciplines \\ Physical Sciences and Mathematics | Physics \\ Comments \\ Suggested Citation: \\ Guasto, J.S., A.S. Ross and J.P. Gollub. (2010). "Hydrodynamic irreversibility in particle suspensions with \\ nonuniform strain." Physical Review E 81, 061401. \\ (C) The American Physical Society \\ http://dx.doi.org/10.1103/PhysRevE.81.061401
}




\title{
Hydrodynamic irreversibility in particle suspensions with nonuniform strain
}

\author{
Jeffrey S. Guasto, ${ }^{1}$ Andrew S. Ross, ${ }^{1}$ and J. P. Gollub ${ }^{1,2}$ \\ ${ }^{1}$ Department of Physics, Haverford College, Haverford, Pennsylvania 19041, USA \\ ${ }^{2}$ Department of Physics, University of Pennsylvania, Philadelphia, Pennsylvania 19104, USA \\ (Received 29 December 2009; revised manuscript received 2 March 2010; published 10 June 2010)
}

\begin{abstract}
A dynamical phase transition from reversible to irreversible behavior occurs when particle suspensions are subjected to uniform oscillatory shear, even in the Stokes flow limit. We consider a more general situation with nonuniform strain (e.g., oscillatory channel flow), which is observed to exhibit markedly different dynamics. The onset of irreversibility is delayed, and occurs simultaneously across the entire channel. This behavior is only partially explained by self-organization and shear-induced migration. The onset is accompanied by longrange correlated particle motion even at the channel center, where the strain is negligible; this motion prevents the system from evolving into a reversible state.
\end{abstract}

DOI: 10.1103/PhysRevE.81.061401

PACS number(s): 83.80.Hj, 47.57.E-, 47.15.G-, 47.52.+j

\section{INTRODUCTION}

Suspensions of non-Brownian particles were recently shown to flow irreversibly when subjected to sufficiently high, uniform oscillatory strain [1] even at low Reynolds number (Stokes flows). The significance of this phenomenon is enhanced by the fact that time reversibility is one of the most basic symmetries of physics, and Stokes flows have long been used to demonstrate hydrodynamic reversibility. Furthermore, a distinct threshold strain amplitude was found above which particles fail to return to their original positions upon flow reversal. This system provides one of the few confirmed examples of a nonequilibrium phase transition [2]. Since suspensions are an important class of fluids in chemical, biological, and industrial applications, the appearance of complex dynamics in such flows is significant. These experiments have led to a number of theoretical works aimed at explaining the nonequilibrium phase transition [3-5].

Random, irreversible particle interactions provide a mechanism for particle self-organization, which leads to a divergent relaxation time at the onset of irreversibility. Similarities have been demonstrated between this transition and plastic depinning phenomena [6]. Uniformly sheared particle suspensions can also exhibit self-organized criticality (SOC) [7] when random interactions and gravitational settling compete.

It has not yet been determined how irreversible particle motion develops in suspension flows that are strained inhomogeneously, a far more general situation. In this paper, we consider the onset of irreversible behavior for dense suspensions ( $40 \%$ by volume) in an oscillatory channel flow. Here, the strain is largest near the walls and negligible at the center, so some regions may locally exceed the critical strain amplitude, while others do not. We find that the transition to irreversibility is markedly different compared to the Couette flow case studied previously. Self-organization and shearinduced migration contribute to a delayed, simultaneous onset of critical behavior across the channel. However, nonlocal particle activity generated in high strain regions near the channel walls prevents the system from becoming reversible even where the strain is negligible, i.e., at the channel centerline. In contrast to the Couette flow case, the fluctuation relaxation time does not diverge at the transition.

\section{EXPERIMENTAL METHODS}

Polymethylmethacrylate (PMMA) particles with a diameter $d=220 \pm 10 \mu \mathrm{m}$ are suspended at a bulk average volume fraction $\bar{\phi}=0.4$ in an index of refraction and density matched solvent $\left(\rho=1.18 \mathrm{~g} / \mathrm{cm}^{3}\right)$ with a viscosity $\mu$ $\approx 3000 \mathrm{cP}[2,10,11]$. The large particle size and solvent viscosity render the particles essentially non-Brownian. A small amount of fluorescent dye (Rhodamine $6 \mathrm{G}$ ) is added to the fluid for visualization purposes. The channel consists of a slot (width $2 a=0.5 \mathrm{~cm}$, depth $2 b=1.5 \mathrm{~cm}$, and length $L$ $=15.0 \mathrm{~cm})$ machined into an optically clear, cast acrylic block. The walls are polished to remove tool marks and an acrylic plate is bonded to the block to close the channel. The suspension is driven in an oscillatory manner using a syringe pump with a low viscosity silicone oil. The interface between the oil and suspension occurs well outside the channel test section [Fig. 1(a)].

A laser sheet (514 nm argon ion laser) is used to probe a thin slice $(\approx 100 \mu \mathrm{m})$ in the bulk of the suspension. The interrogation region $(\approx 1 \mathrm{~cm}$ long $)$ is centered along the length of the channel far from the ends at mid-depth. A charge-coupled device (CCD) camera with a long pass filter $(>550 \mathrm{~nm})$ images the fluorescent dye in the interstices of the suspension $[2,12]$ allowing the particles to be tracked [Fig. 1(b)]. The pump is driven in an approximate square wave pattern (see Video1 [13]) with a peak flow rate $Q$ $=1.0 \mathrm{ml} / \mathrm{min}$ corresponding to a maximum Reynolds number of $\operatorname{Re}=\rho Q / 2 b \mu<10^{-3}$, which ensures Stokes flow conditions. Prior to each experiment, the suspension is mixed thoroughly with a magnetic stirrer bar to provide consistent initial conditions.

With the peak flow rate held constant for all experiments, the strain amplitude, which governs the mobility of the particles, is varied by adjusting the volumetric displacement amplitude of the reciprocating pump. The corresponding driving frequency of the pump is in the range $0.028-0.079 \mathrm{~Hz}$. The suspension is imaged once per period at the same frequency driving the pump for 500 cycles to capture net deviations 
(a)

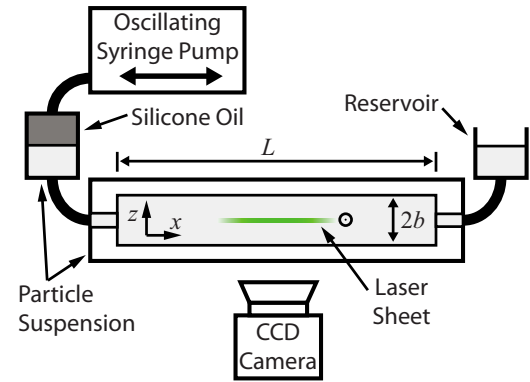

(b)

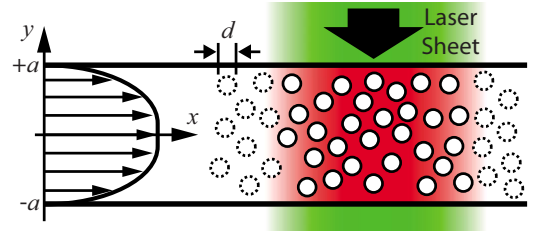

(c)

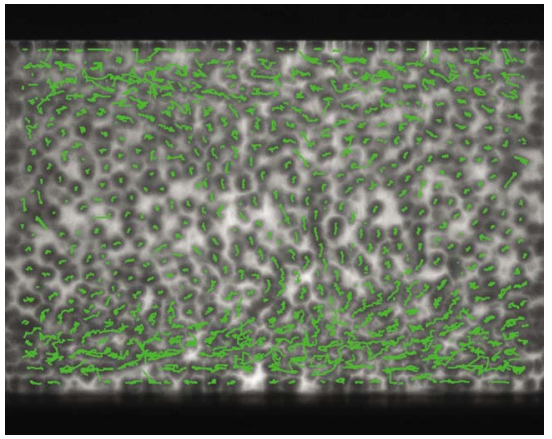

FIG. 1. (Color online) (a) The apparatus uses a syringe pump to drive an oscillating suspension flow. A laser sheet probes a thin plane, which is imaged from below by a CCD camera with a long pass filter. (b) The laser sheet excites fluorescent dye in the interstices between particles, making them visible. (c) Particle tracks overlaid on a multiple exposure image sampled once per period for 35 cycles demonstrates the irreversible behavior especially in high strain regions near the wall.

from reversible particle trajectories measured using particle tracking algorithms [14]. Following the time-lapse imaging, higher speed imaging $(5 \mathrm{fps})$ provides a measurement of the displacement profile, $\delta(y)$, which is used to obtain the strain amplitude, $\gamma=d \delta / d y$ [Fig. 2(a) and inset]. The corresponding steady-state concentration profiles, $\phi(y)$ are also obtained from these measurements and shown in Fig. 2(b). All measurements are averaged about the channel centerline to improve the statistical sampling. The overall strain is parameterized by the largest strain in the system, $\gamma_{0}$, which occurs at the wall.

\section{RESULTS}

Irreversible phenomena such as shear-induced migration have long been observed in suspensions [15-17]. Numerous experiments and simulations have been aimed at understanding particle migration in pressure-driven pipe and channel flows [18-20]. Two regimes of migration are observed in oscillatory pressure-driven systems [21-23], where large oscillation amplitudes cause migration to the centerline, and small amplitudes produce a slight migration toward the

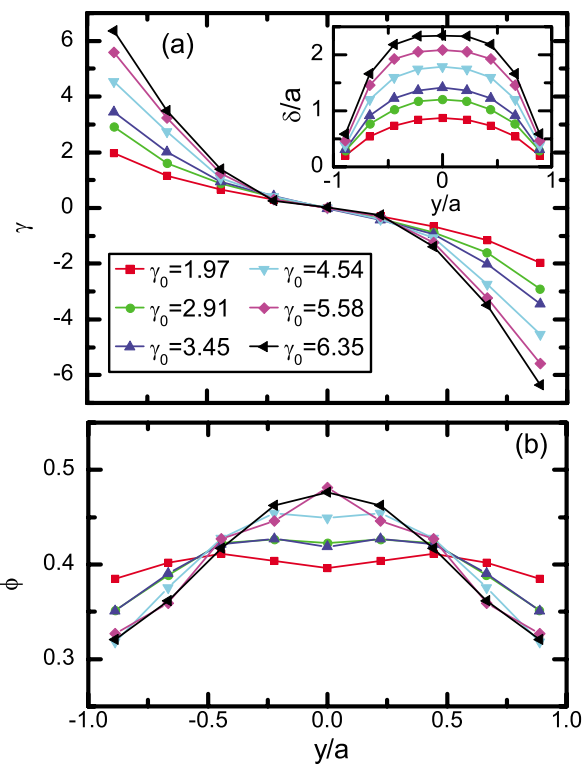

FIG. 2. (Color online) (a) Local strain amplitude estimated from the measured particle displacement profiles (inset). (b) The steadystate particle concentration profiles show significant shear-induced migration at large wall strain amplitudes; the local volume fraction peaks at the channel center.

walls. In the present study, negligible migration is observed for the smallest strain amplitudes tested [Fig. 2(b)]. However, significant migration toward the centerline is observed at large oscillation amplitude. This is accompanied by a wellknown progressive blunting of the displacement profiles shown in Fig. 2(a) (inset) for large strain amplitudes, due to the increased bulk viscosity associated with the locally elevated volume fraction at the channel center. While the bulk effects of these phenomena are well documented, their consequences for particle self-organization and the onset of irreversibility were unknown.

\section{A. Steady-state fluctuations}

The mean square particle displacements (MSDs) measure the degree of irreversibility or particle "activity." We use the stream-wise diffusivity, $D_{x}=\left\langle\Delta x^{2}\right\rangle / 2 N$, (MSD per cycle), and we omit transients occurring in the first $\sim 200$ cycles when considering steady-state measurements. For small wall strain amplitude, the particle trajectory deviations are negligible and thus reversible. As the wall strain increases, we observe a rapid rise of the particle diffusivity in the high strain regions near the walls, and a markedly slower increase near the center $\left(D_{y}\right.$ is somewhat smaller, but shows similar trends) [Fig. 3(a)]. The irreversible nature of the system at large wall strain amplitude is illustrated in Fig. 1(c) by periodically sampled particle tracks over 35 cycles (see also Video2 [13]). We note that the largest fluctuations occur slightly away from the channel walls, as previously observed in steady flows [19].

Irreversible behavior does not occur only where the local strain amplitude surpasses a critical value $\gamma \geq \gamma^{\mathcal{C}}[1,2]$. As the wall strain amplitude is increased, regions in the center of the 

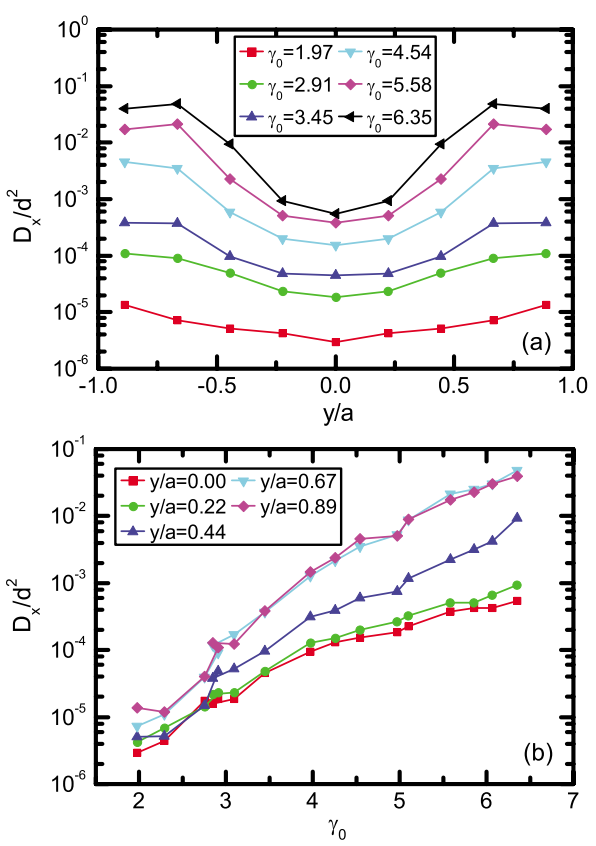

FIG. 3. (Color online) (a) For fixed wall strain amplitude, the particle diffusivity $D_{x}$ is largest near the channel walls, and also rises with the wall strain amplitude for any fixed position. (b) A subset of the same data shows that the development of irreversibility is simultaneous throughout the channel.

channel $(y / a=0)$, where the local strain is negligible, show an increase in diffusivity of two orders of magnitude [Fig. 3(a)]. Although the rate of increase is greater near the wall, the local diffusivity shows a nearly exponential increase with the wall strain amplitude including the centerline, where the local strain amplitude is negligible [Fig. 3(b)].

\section{B. Transition to irreversibility}

The transition to irreversibility in uniformly sheared particle suspensions has been shown to exhibit the characteristics of a continuous phase transition. The characteristic relaxation time $\tau$ for periodically sampled particle fluctuations to reach a steady state was shown to diverge at the onset of irreversibility in the case of uniformly strained suspensions [2]. Following the analysis of Corté et al., the relaxation time is obtained by fitting a time series of the local particle MSDs consisting of 500 cycles from periodically sampled data to $f(t)=\left(f^{0}-f^{\infty}\right) e^{-t / \tau} / t^{\alpha}+f^{\infty}$, where $\alpha=0.6$ and $f^{0}$ and $f^{\infty}$ are the initial and steady values of the particle MSD, respectively [2]. Figure 4(a) (inset) shows sample time series for both low and high strain with fits for $y / a=0.44$ (see also Video2 [13]).

For small wall strains, particles reorganize quickly within a few cycles $(\tau<10$ cycles $)$ and settle into a reversible steady state. As the wall strain is increased, $\tau$ increases abruptly across the entire channel at $\gamma_{0}^{c} \approx 3.4$ indicating a simultaneous onset of irreversible behavior [Fig. 4(a)]. Thus, the local critical strain is elevated at the wall and depressed near the channel center (compared to $\gamma^{\mathcal{C}}=1.6$ for $\bar{\phi}=0.4$ in uniform shear) [1]. We also note that $\tau$ does not diverge, but rather maintains an elevated value $(\approx 130$ cycles $)$ as $\gamma_{0}$ increases further.
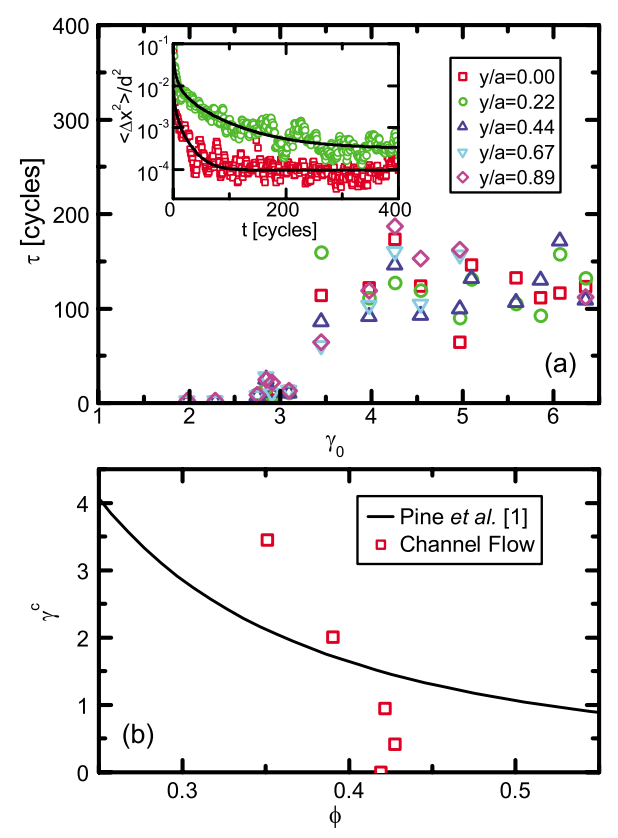

FIG. 4. (Color online) (a) Particle fluctuation relaxation time increases abruptly with wall strain amplitude across the entire channel. Inset: sample mean square displacement time series for $\gamma_{0}$ $=2.8(\square)$ and $\gamma_{0}=4.0(\bigcirc)$ at $y / a=0.44$ with fits. (b) The local critical strain, $\gamma^{\mathcal{C}}$, observed at the global onset $\gamma_{0}^{c} \approx 3.4$, as a function of the local concentration, $\phi$. The much steeper variation here compared with the uniformly strained case demonstrates that concentration variations alone are insufficient to account for the simultaneous criticality across the channel.

The transition to irreversibility is sensitive to the particle concentration [1], and for the conditions tested here, the volume fraction can vary from $30 \%$ at the walls to $50 \%$ in the center of the channel as a result of shear-induced migration [Fig. 2(b)]. The delayed onset of irreversibility can be explained partially by this effect. In Fig. 4(b), we examine the variation in local critical strain amplitude as a function of the local particle concentration (for the single detected critical wall strain, $\gamma_{0}^{c}$ ) and compare it to the critical strain amplitude for the previously studied Couette flow. The much steeper variation here compared with the uniformly strained case shows that concentration variations alone are insufficient to explain the simultaneous criticality across the channel.

\section{Long-range correlated fluctuations}

An important additional factor is the role of irreversible activity near the walls, which appears to influence the core of the channel some distance away. As evidence for this effect, we note that coordinated particle motion is observed in the center of the channel once $\gamma_{0}^{c}$ has been exceeded [Fig. 1(c)] (see also Video2 [13]). The collective fluctuations appearing in the periodically sampled particle motion draw comparison to the dynamical heterogeneity seen in glassy thermal systems [24]. Other works have emphasized the importance of "transient clusters" to the rheology and flow of suspensions, which are due to hydrodynamic interactions between particles that can result in collective particle motion [25]. 


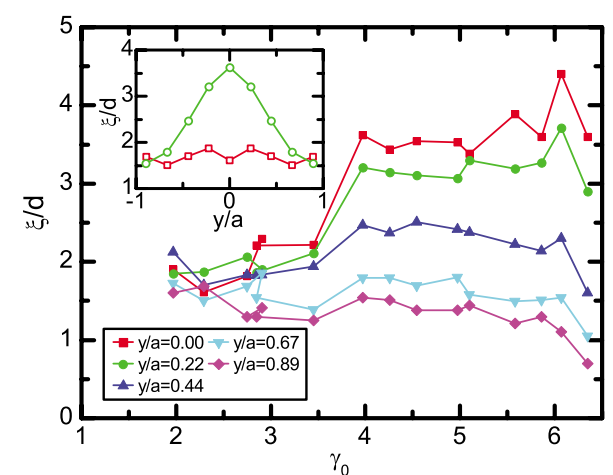

FIG. 5. (Color online) Nondimensional correlation length as a function of wall strain amplitude $\gamma_{0}$ at various positions across the channel. Particle motions in the center of the channel become correlated over a longer range above the critical wall strain amplitude, which indicates that the fluctuations do not arise from local interactions. Inset: nondimensional correlation length as a function of position across the channel for wall strain amplitudes of $\gamma_{0}=2.3$ $(\square)$ and $\gamma_{0}=4.0(\bigcirc)$.

We quantified the observed collective motion in the steady state by a displacement correlation function $g\left(r^{\prime}\right)$ $=\left\langle\Delta \vec{r}_{i} \cdot \Delta \vec{r}_{j}\right\rangle /\left\langle\Delta \vec{r}_{i} \cdot \Delta \vec{r}_{i}\right\rangle$, where $r^{\prime}=\left|\vec{r}_{j}-\vec{r}_{i}\right|$ is the magnitude of the separation between any two particles and $\Delta \vec{r}_{i}$ is the vector displacement of a given particle in the image plane after a given cycle. The angled brackets denote the ensemble average over all particles, and the vector dot product of the particle displacements accounts for comoving particles in any direction within the image plane. The corresponding correlation length is denoted by $\xi$ and is computed from exponential fits of $g\left(r^{\prime}\right)$. The largest measurable correlations are limited by the finite system size.

Below the critical strain amplitude, particle motion in the periodically sampled data is minimal and spatially uncorrelated across the channel [Fig. 5 (inset)]. However, once the critical strain amplitude is surpassed, the correlation length increases in the center, but remains small near the walls [Fig. $5]$. The larger correlation length of the particle motion in the center region implies that these fluctuations originate from irreversible disturbances some distance away rather than from local particle interactions.

\section{CONCLUSION}

We have studied the onset of irreversible motion in particle suspensions in an oscillatory channel flow, where the shear strain is nonuniform. The transition is shown to be quite different from the previously studied Couette flow case in that it does not exhibit a divergent relaxation time. Strikingly, the transition is delayed to higher wall strain amplitude, and occurs simultaneously across the channel, even where the local strain is negligible. We find that selforganization and shear-induced migration alone cannot explain the simultaneous onset. The transition to irreversible dynamics is not governed purely by the local strain, but depends also on disturbances generated some distance away.

It would be interesting to know how our results might be affected by small amounts of Brownian motion, as in colloidal systems [8,9]. However, it is not known how the transition to irreversibility might be modified even in the simplest case of uniform shear.

This work is an important step in understanding the coupled behavior of irreversible particle motion (activity), local particle concentration, and imposed strain [4]. While the nature of the collective particle motion in the periodically sampled case is reminiscent of the dynamical heterogeneity observed in glassy thermal systems [24], the origin of the nonlocality is not well understood. Some researchers have emphasized the role of transient aggregates due to hydrodynamic interactions, but friction or mechanical contacts between particles can also result in force chains which transmit nonlocal activity many diameters away [26]. Many challenges remain including consideration of nonlocal effects in models of the transition, the possible role of finite system size, and exploration of other materials such as polydisperse or soft suspensions.

\section{ACKNOWLEDGMENTS}

We thank David Pine and Laurent Corté for helpful discussions, and Bruce Boyes for technical assistance. This work was supported by NSF Grant No. DMR-0803153.
[1] D. J. Pine, J. P. Gollub, J. F. Brady, and A. M. Leshansky, Nature (London) 438, 997 (2005).

[2] L. Corté, P. M. Chaikin, J. P. Gollub, and D. J. Pine, Nat. Phys. 4, 420 (2008).

[3] G. Düring, D. Bartolo, and J. Kurchan, Phys. Rev. E 79, 030101(R) (2009).

[4] G. I. Menon and S. Ramaswamy, Phys. Rev. E 79, 061108 (2009).

[5] I. Santamaría-Holek, G. Barrios, and J. M. Rubi, Phys. Rev. E 79, 031201 (2009).

[6] C. Reichhardt and C. J. OlsonReichhardt, Phys. Rev. Lett. 103, 168301 (2009).

[7] L. Corté, S. J. Gerbode, W. Man, and D. J. Pine, Phys. Rev.
Lett. 103, 248301 (2009).

[8] M. Frank, D. Anderson, E. R. Weeks, and J. F. Morris, J. Fluid Mech. 493, 363 (2003).

[9] L. Isa, R. Besseling, and W. C. K. Poon, Phys. Rev. Lett. 98, 198305 (2007).

[10] G. P. Krishnan, S. Beimfohr, and D. T. Leighton, J. Fluid Mech. 321, 371 (1996).

[11] V. Breedveld, D. van den Ende, A. Tripathi, and A. Acrivos, J. Fluid Mech. 375, 297 (1998).

[12] J. C. Tsai and J. P. Gollub, Phys. Rev. E 70, 031303 (2004).

[13] See supplementary material at http://link.aps.org/supplemental/ 10.1103/PhysRevE.81.061401 for videos.

[14] N. T. Ouellette, H. Xu, and E. Bodenschatz, Exp. Fluids 40, 
301 (2006).

[15] D. Leighton and A. Acrivos, Chem. Eng. Sci. 41, 1377 (1986).

[16] D. Leighton and A. Acrivos, J. Fluid Mech. 181, 415 (1987).

[17] J. J. Stickel and R. L. Powell, Annu. Rev. Fluid Mech. 37, 129 (2005).

[18] R. E. Hampton, A. A. Mammoli, A. L. Graham, N. Tetlow, and S. A. Altobelli, J. Rheol. 41, 621 (1997).

[19] M. K. Lyon and L. G. Leal, J. Fluid Mech. 363, 25 (1998).

[20] P. R. Nott and J. F. Brady, J. Fluid Mech. 275, 157 (1994).

[21] J. E. Butler, P. D. Majors, and R. T. Bonnecaze, Phys. Fluids
11, 2865 (1999).

[22] J. F. Morris, Phys. Fluids 13, 2457 (2001).

[23] K. Yapici, R. L. Powell, and R. J. Phillips, Phys. Fluids 21, 053302 (2009).

[24] B. Cui, B. Lin, and S. A. Rice, J. Chem. Phys. 114, 9142 (2001).

[25] P. Mills and P. Snabre, J. Phys. II 5, 1597 (1995).

[26] T. S. Majmudar and R. P. Behringer, Nature (London) 435, 1079 (2005). 